\title{
Sudden Onset of Lower Abdominal Pain Without Peritonitis or Ileus
}

\author{
Stefania Scarlini, Marco Gandolfo, Antonello Pietrangelo \\ Unit of Internal Medicine 2, University Hospital Policlinico of Modena, University of Modena and Reggio Emilia, Modena, Italy
}

Received: 10/08/2016

Accepted: $22 / 08 / 2016$

Published: 14/09/2016

How to cite this article: Scarlini S, Gandolfo M. Pietrangelo A. Sudden onset of lower abdominal pain without peritonitis or ileus. EJCRIM 2016;3:doi:10.128 90/2016_000444.

Conflicts of Interests: The Authors declare that there are no competing interests.

This article is licensed under a Commons Attribution Non-Commercial 4.0 License

\section{ABSTRACT}

A patient presented with sudden, unexplained lower abdominal pain without peritonism or signs of infection or inflammatory reaction, but with recent bloody stools and a history of radiation therapy, diabetes and immunosuppression. Plain abdominal x-ray showed only air-fluid levels and air distention of the colon, but a later abdominal CT scan revealed extensive gas gangrene of the colon. The patient's clinical status rapidly worsened. Elective surgical rectosigmoid debridement did not prevent the patient's death. In conclusion, the diagnosis of 'spontaneous' life-threatening gas gangrene requires a high degree of clinical suspicion and allows life-saving surgical intervention.

\section{LEARNING POINTS}

- Sudden and rapidly worsening lower abdominal pain without peritonitis or ileus can indicate gas gangrene from distal bowel perforation.

- Bloody stools or other symptoms and/or procedures reported previously and apparently resolved could indicate hidden perforation.

- Diagnostic gas detection, clinically or radiologically, occurs too late to prevent fatal consequences, so early signs of infection and inflammation should be sought for and evaluated as early surgery is life saving.

\section{KEYWORDS}

Gas gangrene, Clostridium septicum, acute abdominal pain

\section{INTRODUCTION}

Gas gangrene is associated with perforating trauma. However, the lower bowel can perforate spontaneously and anaerobes from the intestinal flora can cause life-threatening soft tissue gangrene. Spontaneous perforation, often due to a weakened intestinal wall, is a rare and severe occurrence with high mortality due to the lack of obvious clinical manifestations and rapid development of systemic toxicity. A high level of suspicion is essential for early diagnosis and immediate treatment.

\section{CASE PRESENTATION}

A 60-year-old man with a history of diabetes, pulmonary small cell carcinoma being treated with carboplatin-etoposide and previous radiotherapy for prostate cancer, complained of the onset of stabbing and diffuse, non-irradiated, abdominal pain. He mentioned having bloody stools 3 days previously that had spontaneously resolved. When he visited his doctor 2 days before hospitalization, he was asymptomatic except for slight haemorrhoidal congestion. On admission he was febrile $\left(38.5^{\circ} \mathrm{C}\right)$ and haemodynamically stable but with acute pain in the lower abdominal quadrants without peritonism.

Peristalsis was torpid but the bowel was open. Abdominal x-ray showed a colon distended with air and fluid. Laboratory tests showed 


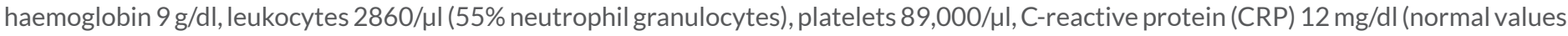
$<0.5 \mathrm{mg} / \mathrm{dl}$ ), CPK $470 \mathrm{U} / \mathrm{I}$ (normal values < $350 \mathrm{U} / \mathrm{I}$ ) and plasma creatinine $2.3 \mathrm{mg} / \mathrm{dl}$. Due to the suspicion of infection, empirical therapy with piperacillin-tazobactam was started after sample collection for blood cultures. Pain sedation required the use of morphine. After $6 \mathrm{~h}$ the patient developed hypotension, tachycardia and chest pain. The ECG and troponin levels were diagnostic for non-ST elevation myocardial infarction (NSTEMI). Hemogasanalysis showed pH 7.19, HCO3 17 and lactate 30, indicating severe metabolic acidosis. Intravenous fluid resuscitation and teicoplanin infusion were started. The patient was transferred to the ICU where he became unconscious. A total body CT scan was performed and showed a diffuse gas gangrene starting from the rectum and extending to the thoracic muscles (Fig. 1). A surgical rectosigmoid resection was immediately performed but the patient died $8 \mathrm{~h}$ after surgery. Blood and intestinal tissue cultures were positive for Clostridium septicum.
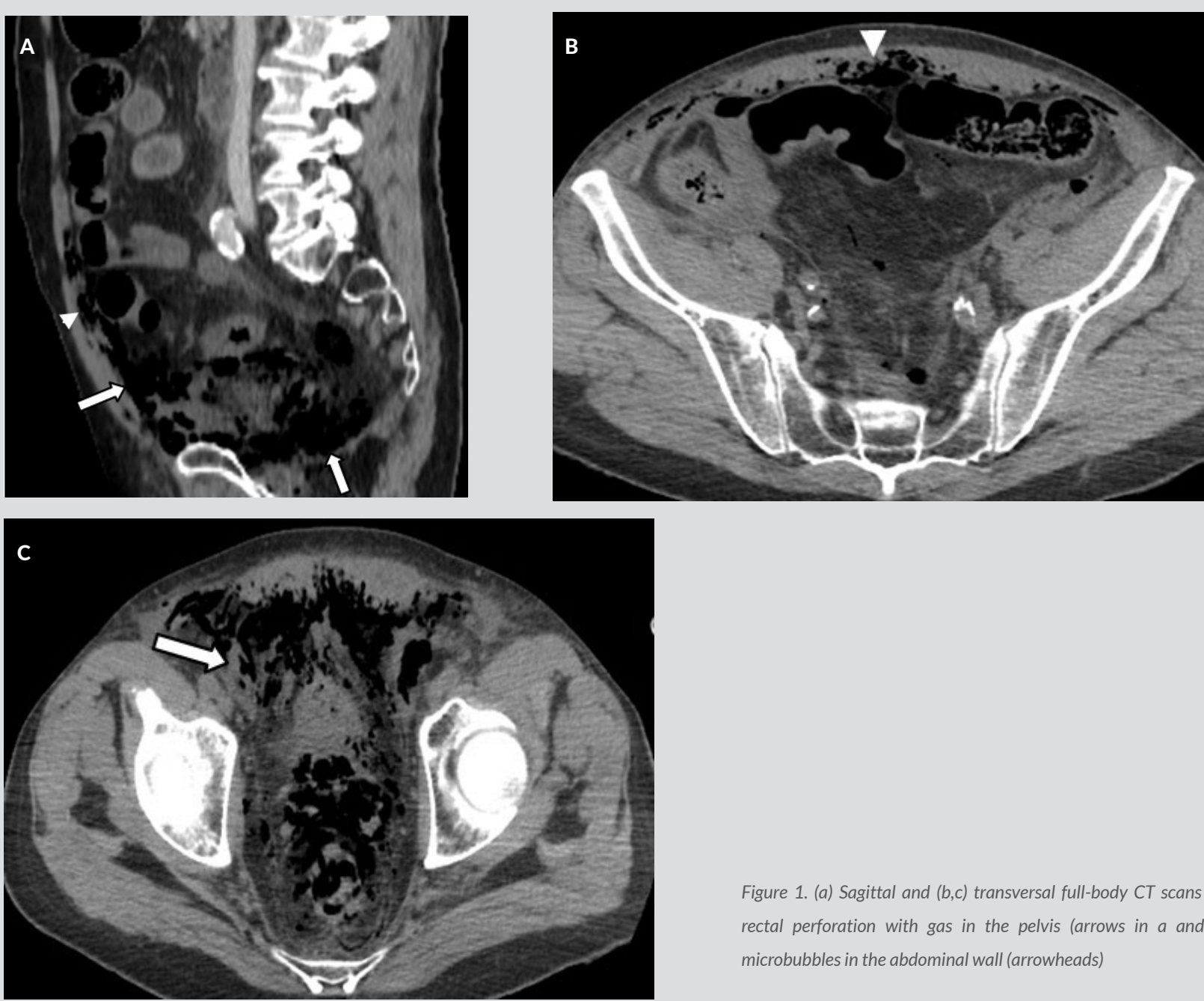

Figure 1. (a) Sagittal and (b,c) transversal full-body CT scans showing rectal perforation with gas in the pelvis (arrows in $a$ and c) with microbubbles in the abdominal wall (arrowheads)

\section{DISCUSSION}

Gas gangrene, usually caused by Clostridium septicum, is a severe infection leading to muscular and soft tissue necrosis. It is generally associated with perforating trauma but, in rare cases (e.g. patients with bowel malignancy, radiation therapy, leukaemia, diabetes and immunosuppression) can occur without apparent perforation. Signs of systemic toxicity develop rapidly, followed by shock and multiple organ failure. Death usually occurs within 24-48 h after symptom onset. Early diagnosis is essential but difficult: the lower intestinal 
perforation is not readily apparent, while gas in tissues, detectable clinically as crepitus, or radiologically, develops late, although a history of previous procedures, like enemas etc., can give rise to suspicion ${ }^{[1-2]}$. The most important alert sign is acute and unexplained abdominal pain that should immediately call for appropriate imaging (CT or MRI). After diagnosis, early antibiotic treatment with clindamycin 600-900 mg/ kg every $8 \mathrm{~h}$ plus penicillin 2-4 million units every 4-6 h and aggressive surgical debridement are essential[3].

In our case, the bloody stools seen a few days previously, which apparently did not cause concern and were unexplained (although initially attributed to haemorrhoids), may well have been an early sign of rectal wall perforation. In fact, a number of factors, in particular radiation treatment for prostate cancer and immunosuppression therapy, may have weakened and fissured the distal intestinal wall, favouring access of Gram-negative bacteria and anaerobes to peri-rectal fat and leading to soft tissue gangrene. The sudden lower abdominal pain is the second key diagnostic element. In our case, lack of peritonism, due to the extra-peritoneal site of intestinal perforation, and immediate and aggressive pain treatment with morphine that rapidly reduced the key alarming symptom, affected our clinical judgement and delayed further imaging evaluation. In this context, a sharp pain could also suggest a dissecting aortic aneurism and prompt abdominal ultrasound evaluation. The third important clinical element of this case was the impending septic shock. Neutropenia, renal failure and a generalized inflammatory reaction (which may have also caused troponin elevation, initially attributed to NSTEMI) may all be signs of Gram-negative sepsis or anaerobic infection. The empirical antibiotic therapy was clearly ineffective for controlling the infection sustained by the Clostridium in our immunodepressed patient who died shortly after surgery due to severe sepsis.

\section{CONCLUSION}

Acute and unexplained abdominal pain can be the only early manifestation of spontaneous gas gangrene. Lack of peritonism should not mean surgical evaluation is postponed and the patient should be immediately investigated with a diagnostic radiological work-up (CT or MRI). Aggressive surgical debridement remains the only life-saving strategy.

\section{REFERENCES}

1. Stevens DL, Bisno AL, Chambers HF, Everett ED, Dellinger P, Goldstein EJC, et al. Practice guidelines for the diagnosis and management of skin and soft-tissue infections. Clin Infect Dis 2005;41:1373-1406.

2. Mishra SP, Singh S, Gupta SK. Necrotizing soft tissue infections: surgeon's prospective. J Inflamm 2013;2013:1-7.

3. Hartel M, Kutup A, Gehl A, Zustin J, Grossterlinden LG, Rueger JM, et al. Foudroyant course of an extensive Clostridium septicum gas gangrene in a diabetic patient with occult carcinoma of the colon. Orthopedics 2013;2013:1-5. 